\title{
Optimization models for flexgrid elastic optical networks
}

\author{
Mateusz Żotkiewicz ${ }^{1 \star}$, Michał Pióro ${ }^{1}$, Marc Ruiz ${ }^{2}$, Mirosław Klinkowski ${ }^{3}$, Luis Velasco ${ }^{2}$ \\ 1 Warsaw University of Technology, Warsaw, Poland \\ ${ }^{2}$ Universitat Politècnica de Catalunya, Barcelona, Spain \\ ${ }^{3}$ National Institute of Telecommunications, Warsaw, Poland \\ *mzotkiew@tele.pw.edu.pl
}

\begin{abstract}
In the paper we present integer programming (IP) optimization models for flexgrid elastic optical networks (EON). We consider several different basic assumptions regarding flexibility of EON that lead to a variety of IP formulations differing in precision and complexity. As usual, detailed models aiming at precisely describing technological aspects of EON suffer from tractability issues resulting from their greater complexity and have to be reasonably simplified. To achieve this, we consider cases where the bandwidth is divided into predefined slots, cases where the bandwidth is continuous and can be divided between demands with no restrictions, cases where a list of predefined paths is available, and finally cases where all the paths are indirectly taken into account. We present both compact and non-compact formulations. The non-compact formulations are accompanied with brief description of the dedicated column generation algorithms.
\end{abstract}

Keywords: flexgrid optical network; routing and spectrum allocation; mathematical modeling

\section{INTRODUCTION}

The most promising solution to deal with huge data traffic demands in communication networks is to use recent technologies in flexible optical networking, and in particular the flexible grid (flexgrid) technology specified in the ITU-T G.694.1 Recommendation [1]. In the flexgrid solution, the frequency spectrum of an optical fiber link is divided into narrow frequency slots. Any sequence of consecutive slots can be used as one channel, and such a channel can be switched in the network nodes to create a lightpath. Thus, a lightpath is determined by a route and a selected channel. More details on flexible optical network architectures can be found in [2].

In flexgrid optical networks, the problem of establish lightpaths for a set of end-to-end demands that compete for spectrum resources is called the Routing and Spectrum Allocation (RSA) problem. RSA consists of assigning a lightpath to each demand. Each such lightpath is routed in a network graph and is assigned a contiguous fraction of frequency spectrum reserved on the route. Clearly, frequencies assigned to individual lightpaths cannot overlap on network links. Moreover, it is commonly assumed that the same piece of the spectrum is used on all the links traversed by a lightpath.

Several alternative Mixed Integer Programming (MIP) formulations of the RSA problem can be found in the literature [3]-[6]. In this paper we present the state-of-the-art of this field.

\section{OPTIMIZATION MODElS}

The whole notation used in this section is gathered in Table I. All the problems, to be presented, are RSA problems with an objective function minimizing the total sum of lengths of all utilized lightpaths. The objective function can be easily exchanged, upgraded, or even omitted, as the main goal of the models presented in this paper is to define sets of constraints that describe feasible solution to the RSA problem.

\subsection{Link-path formulation involving channels - LPC}

The first presented model can be considered as a classical approach. In the model a notion of a lightpath is used. A lightpath is understood as a pair: route and channel. The route is a path through a network from a source node to a termination node of a demand, while the channel is a set of slots assigned to the lightpath. In this formulation it is assumed that sets of possible lightpaths $\mathcal{L}(d)$ for each demand are given, thus the problem simplifies to selecting one of those lightpaths for each demand in such a way that there are no two demand that use the same slot on the same link. The formal formulation is given below. Variables in brackets, i.e, $\lambda_{d}$ and $\pi_{e s}$, are dual variables. A similar formulation can be found in [6].

This work has been partially funded by the FP7 project IDEALIST (grant agreement no. 317999). M. Żotkiewicz was supported by the EC European Social Fund through the Warsaw University of Technology Development Programme, M. Pióro and M. Klinkowski were supported by National Science Center (Poland) under grants 2011/01/B/ST7/02967 and 2011/01/D/ST7/05884, respectively. M. Ruiz and L. Velasco were supported by the Spanish Science Ministry through TEC2011-27310 ELASTIC. 
TABLE I

NOTATION

$\begin{array}{ll}\text { Indices: } & \\ e & \text { link } \\ d & \text { demand } \\ p & \text { route }\end{array}$

Sets:

$\mathcal{E} \quad$ set of links

$\mathcal{V} \quad$ set of nodes

$\mathcal{D} \quad$ set of demands

$\mathcal{S} \quad$ set of spectrum slots, $\mathcal{S}=\{1,2, \ldots, S\}$

$\delta^{+}(v) \quad$ set of links leaving node $v$

$\mathcal{C}$ channel; a set of slots

\section{Constants:}

$S \quad$ number of slots

$c(l) \quad$ length of lightpath $l$

$h(d) \quad$ volume of demand $d$

$t(d) \quad$ sink of demand $d$ node

lightpath

spectrum slot

Variables:

$x_{d l} \quad$ binary, $x_{d l}=1$ when demand $d$ uses lightpath $l ; x_{d l}=0$ otherwise

$x_{d p} \quad$ binary, $x_{d p}=1$ when demand $d$ uses route $p ; x_{d p}=0$ otherwise

$y_{d s} \quad$ binary, $y_{d s}=1$ when demand $d$ uses slot $s ; y_{d s}=0$ otherwise

$a_{d s} \quad$ binary, $a_{d s}=1$ when demand $d$ uses slots that are greater than $s ; a_{d s}=0$ otherwise

$b_{d s} \quad$ binary, $b_{d s}=1$ when demand $d$ uses slots that are smaller than $s ; b_{d s}=0$ otherwise

$a_{d} \quad$ first slot used by demand $d$, i.e., slots $0, \ldots, a_{d}-1$ are not used by demand $d$

$b_{d} \quad$ last slot used by demand $d$, i.e., slots $b_{d}+1, \ldots, S$ are not used by demand $d$

$c_{d d^{\prime}} \quad$ binary, $c_{d d^{\prime}}=1$ if $a_{d} \leq a_{d^{\prime}} ; c_{d d^{\prime}}=0$ otherwise

$x_{d e} \quad$ binary, $x_{d e}=1$ when demand $d$ uses link $e ; x_{d e}=0$ otherwise

$$
\begin{array}{r} 
\\
{\left[\lambda_{d}\right] \quad \min \sum_{d \in \mathcal{D}} \sum_{l \in \mathcal{L}(d)} c(l) x_{d l}} \\
\sum_{l \in \mathcal{L}(d)} x_{d l}=1 \\
\sum_{d \in \mathcal{D}} \sum_{l \in \mathcal{Q}(d, e, s)} x_{d l} \leq 1 \\
x_{d l} \in\{0,1\}
\end{array}
$$

Equation (1b) assures that each demand will use one and only one lightpath from a set of allowable lightpaths, while (1c) assures that there are no collisions of the assigned resources, i.e., there are no two lightpaths in a network that use the same slot on the same link. Finally (1d) assures that the variables are binary.

In order to solve the problem to optimality, either sets $\mathcal{L}(d)$ have to contain all allowable lightpaths for each demand or a column generation techniques has to be applied. The former approach is impractical. On the other hand, the latter approach can be successfully applied. To find a possibly profitable new lightpath in a network for each demand $d$ and each channel $\mathcal{C}=\left\{s_{1}, s_{2}, \ldots, s_{n}\right\}$ satisfying demand $d$, the shortest path with respect to weights of links equal to $1+\sum_{s \in \mathcal{C}} \pi_{e s}$ has to be found. If a cost of this path is smaller than $\lambda_{d}$, then a lightpath using the computed shortest path and the assumed channel should be added to $\mathcal{L}(d)$.

\subsection{Link-path formulation involving slots - LPS}

In Section 2.1 sets of all allowable lightpaths for each demand were used, thus in the formulation a possible number of variables is huge - a variable is needed for each possible pair of a route and a channel. We address this problem in the next formulation, where a notion of a lightpath is abandoned, and is replaced by a notion of routes and slot assignments. Therefore, the resources used by a demand are now: a route defined by variables $x_{d p}$ and utilized slots defined by variables $y_{d s}$.

In order to successfully define a problem we need set $\mathcal{R}$ of pairs of routes for different demands that share at least one link. Formally the set is defined as $\mathcal{R}=\left\{\left(d, d^{\prime}, p, p^{\prime}\right): d, d^{\prime} \in \mathcal{D}, d \neq d^{\prime}, p \in \mathcal{P}(d), p^{\prime} \in\right.$ $\left.\mathcal{P}\left(d^{\prime}\right), p \cap p^{\prime} \neq \emptyset\right\}$. Additionally we assume that slots are ordered, i.e., $\mathcal{S}=\{1,2, \ldots, S\}$. Those assumptions allow us to formulate the problem as follows. Again variables in brackets are dual variables. 


$$
\begin{aligned}
& \min \sum_{d \in \mathcal{D}} \sum_{p \in \mathcal{P}(d)} c(p) x_{d p} \\
& {\left[\lambda_{d}\right] \quad \sum_{p \in \mathcal{P}(d)} x_{d p}=1} \\
& {\left[\gamma_{d d^{\prime} p p^{\prime}}\right] \quad x_{d p}+y_{d s}+x_{d^{\prime} p^{\prime}}+y_{d^{\prime} s} \leq 3} \\
& a_{d s} \geq a_{d s+1} \\
& b_{d s} \geq b_{d s-1} \\
& y_{d s}+a_{d s}+b_{d s}=1 \\
& B \sum_{s \in \mathcal{S}} y_{d s} \geq h_{d} \\
& y_{d s}, a_{d s}, b_{d s} \in\{0,1\} \\
& x_{d p} \in\{0,1\} \\
& d \in \mathcal{D} \\
& s \in \mathcal{S},\left(d, d^{\prime}, p, p^{\prime}\right) \in \mathcal{R} \\
& d \in \mathcal{D}, s \in \mathcal{S} \backslash\{S\} \\
& d \in \mathcal{D}, s \in \mathcal{S} \backslash\{1\} \\
& d \in \mathcal{D}, s \in \mathcal{S} \\
& d \in \mathcal{D} \\
& d \in \mathcal{D}, s \in \mathcal{S} \\
& d \in \mathcal{D}, p \in \mathcal{P}(d)
\end{aligned}
$$

Equation ( $2 \mathrm{~b})$ assures that each demand will use one route for its lightpath. Knowing the route $\left(x_{d p}\right)$ and the channel assignment $\left(y_{d s}\right)$ it is possible to assure that there will be no demands using the same resources. This condition is enforced by (2c). In order to assure that slots assigned to a demand are consecutive slots auxiliary variables $a_{d s}$ and $b_{d s}$ are used, both meaning that slot $s$ is not used by demand $d$. Equation (2f) ensures that for each demand $d$ and slot $s$ exactly one of variables $a_{d s}, b_{d s}$, or $y_{d s}$ is equal 1 . Constraint (2d) assures that if for slot $s$ variable $a_{d s}$ is selected, then for each slot $s^{\prime}$ with a lower identification number variable $a_{d s^{\prime}}$ has to be also selected, while (2e) assures that if for slot $s$ variable $b_{d s}$ is selected, then for each slot $s^{\prime}$ with a greater identification number variable $b_{d s^{\prime}}$ has to be also selected. Summing up, constraints eqrefeq:LPS:a $i$ a, (2e), and (2f) assure that channels are formed by consecutive slots. The requested size of the channel is controlled by $(2 \mathrm{~g})$. Finally, $(2 \mathrm{~h})$ and $(2 \mathrm{i})$ assure that variables are binary.

To solve the problem to optimality either sets $\mathcal{P}(d)$ have to contain all allowable routes for each demand or a column generation techniques has to be applied. The former approach is impractical. Unfortunately, in this case, also the latter approach is not recommended. The reason is that not only variables $x_{d p}$ but also constraints (2c) have to be added to the model. Reduced costs of possibly new routes depend on dual variables $\gamma_{d d^{\prime} p p^{\prime}}$ for constraints that have to be also generated. Such a situation leads to cases when adding any new route can lead to infeasibility of a current dual solution, as long as $\lambda_{d}$ is greater than zero. It makes the approach impractical.

\subsection{Link-path formulation with continuous spectrum - LPCS}

According to the standardization [1] a set of allowable slots is given and finite. However, its size is large enough that formulations involving variables indexed by single slots can grow to prohibitive sizes. Therefore, it is worth considering to work just on identification number $a_{d}$ of a starting slot of a channel and identification number $b_{d}$ of an ending slot of a channel. Knowing that a channel has to be formed by consecutive slots, this approach can save orders of magnitude of memory space while formulating the considered problem. Additionally, in order to formulate the problem, binary variable $c_{d d^{\prime}}$ is needed. The variable indicates if identification numbers of slots used by demand $d$ are smaller than identification numbers of slots used by demand $d^{\prime}$ or the opposite. A similar formulation can be found in [4].

$$
\begin{array}{rr}
\min \sum_{d \in \mathcal{D}} \sum_{p \in \mathcal{P}(d)} c(p) x_{d p} & \\
\sum_{p \in \mathcal{P}(d)} x_{d p}=1 & d \in \mathcal{D} \\
\left(x_{d p}+x_{d^{\prime} p^{\prime}}+c_{d d^{\prime}}-3\right) S<a_{d^{\prime}}-b_{d} & \left(d, d^{\prime}, p, p^{\prime}\right) \in \mathcal{R} \\
\left(x_{d p}+x_{d^{\prime} p^{\prime}}-c_{d d^{\prime}}-2\right) S<a_{d}-b_{d^{\prime}} & \left(d, d^{\prime}, p, p^{\prime}\right) \in \mathcal{R} \\
B\left(b_{d}-a_{d}+1\right) \geq h_{d} & d \in \mathcal{D} \\
a_{d}, b_{d} \in\{1,2, \ldots, S\} & d \in \mathcal{D} \\
c_{d d^{\prime}} \in\{0,1\} & d, d^{\prime} \in \mathcal{D} \\
x_{d p} \in\{0,1\} & d \in \mathcal{D}, p \in \mathcal{P}(d)
\end{array}
$$

Equation ( $3 b$ ) assures that each demand will use one route for its lightpath. The fact that two demands cannot use the same slot on the same link is enforced by (3c) and (3c). In case two demands $d$ and $d^{\prime}$ are using routes 
that have at least one link in common two scenarios can happen. First, if $d$ is supposed to use slots of lower identification numbers $\left(c_{d d^{\prime}}=1\right)$, then $(3 \mathrm{c})$ is active, and the constraint reduces to $b_{d}<a_{d^{\prime}}$. In other case, constraint (3d) is active, and it reduces to $b_{d^{\prime}}<a_{d}$. Notice that in both (3c) and (3d) constant $S$ is used as so called big $M$. The requested size of the channel is controlled by (3e). Finally, constraints (3f), (3g), and (3h) assure that variables are integral $\left(a_{d}\right.$ and $\left.b_{d}\right)$ or binary $\left(c_{d d^{\prime}}\right.$ and $\left.x_{d p}\right)$.

Unfortunately, the same remarks as in Section 2.2 concerning solving the problem to optimality applies in this section, i.e., the problem can be effectively solved only if allowable sets of routes are given in advance.

\subsection{Node-link formulation involving slots - NLS}

All the formulations given above suffer from one drawback-they are not compact, thus solving them to optimality involves either using huge sets of allowable routes or applying column generation techniques. This problem is addressed by a formulation presented in this section. The formulation is similar to the formulation of Section 2.2. The only difference is the way routes are handled. In the previous approach a variable was defined for each demand-route pair. In this approach a variable is defined for each demand-link pair.

$$
\begin{array}{r}
\sum_{e \in \delta^{+}(s(d))} x_{d e}-\sum_{e \in \delta^{-}(s(d))} x_{d \in \mathcal{D}} \sum_{e \in \mathcal{E}} x_{d e} \\
\sum_{e \in \delta^{+}(v)} x_{d e}-\sum_{e \in \delta^{-}(v)} x_{d e}=0 \\
x_{d e}+y_{d s}+x_{d^{\prime} e}+y_{d^{\prime} s} \leq 3 \\
a_{d s} \geq a_{d s+1} \\
b_{d s} \geq b_{d s-1} \\
y_{d s}+a_{d s}+b_{d s}=1 \\
B \sum_{s \in \mathcal{S}} y_{d s} \geq h_{d} \\
d \in \mathcal{D}, v \in \mathcal{V} \backslash\{s(d), t(d)\} \\
s \in \mathcal{S}, d, d^{\prime} \in \mathcal{D}, e \in \mathcal{E} \\
d \in \mathcal{D}, s \in \mathcal{S} \backslash\{S\} \\
d \in \mathcal{D}, s \in \mathcal{S} \backslash\{1\} \\
d \in \mathcal{D}, s \in \mathcal{S} \\
\quad d \in \mathcal{D} \\
y_{d s}, a_{d s}, b_{d s} \in\{0,1\} \\
x_{d e} \in\{0,1\} \\
d \in \mathcal{D}, s \in \mathcal{S} \\
d \in \mathcal{D}, e \in \mathcal{E} .
\end{array}
$$

The only novel constraints of (4) are (4c), (4b), and (4d). The first two express the Kirchhoff's law for flows. Each route is now a flow of unit size from $s(d)$ to $t(d)$. Constraint $(4 \mathrm{~d})$ assures that reserved resources are not shared by not allowing two demands to use the same link while simultaneously using the same slot. The rest of the constraints are identical to constraints of (2).

This formulation is compact, so it can be solved to optimality using the standard branch-and-bound approach.

\section{Conclusion}

In the paper we presented four different MIP formulations of the RSA problem that differ in their complexity and applicability. Formulation LPC should be used when a set of lightpaths is given or all lightpaths should be taken into account. However, the latter case would require using branch-and-price techniques. An alternative approach is NLS, which indirectly takes all lightpaths into account. It is less efficient than LPC. However, it is much simpler to implement. Remaining two approaches should be used when a set of routes is defined. LPS should be used for a small number of slots, while LPCS should be used for a large number of slots.

\section{REFERENCES}

[1] "Spectral grids for WDM applications: DWDM frequency grid," ITU-T G.694.1 (ed. 2.0), 2012.

[2] M. Jinno, H. Takara, B. Kozicki, Y. Tsukishima, Y. Sone, and S. Matsuoka, "Spectrum-efficient and scalable elastic optical path network: Architecture, benefits, and enabling technologies," IEEE Commun. Mag., vol. 47, no. 11, pp. 66-73, 2009.

[3] M. Klinkowski and K. Walkowiak, "Routing and spectrum assignment in spectrum sliced elastic optical path network," IEEE Commun. Lett., vol. 15, no. 8, pp. 884 -886, august 2011.

[4] K. Christodoulopoulos, I. Tomkos, and E. A. Varvarigos, "Elastic bandwidth allocation in flexible OFDMbased optical networks," IEEE J. Lightw. Technol., vol. 29, no. 9, pp. 1354 -1366, may 2011.

[5] Y. Wang, X. Cao, Q. Hu, and Y. Pan, "Towards elastic and fine-granular bandwidth allocation in spectrumsliced optical networks," IEEE J. Opt. Commun. Netw., vol. 4, no. 11, pp. 906 -917, nov. 2012.

[6] L. Velasco, M. Klinkowski, M. Ruiz, and J. Comellas, "Modeling the routing and spectrum allocation problem for flexgrid optical networks," Photonic Netw. Commun., vol. 24, pp. 177-186, 2012. 